\title{
Enfermería: fomento de la autoestima en padecimientos neuróticos en el adulto mayor ${ }^{1}$
}

Giovanna Enríquez Gutiérrez ${ }^{2}$

Silvia Nájera Aguirre ${ }^{3}$

Institución: Universidad Autónoma de Ciudad Juárez, México

\section{CÓMO CITAR}

Enríquez, G. y Nájera, S. (Abril, 2014) Enfermería: fomento de la autoestima en padecimientos neuróticos en el adulto mayor. Rev. Actual de Costa Rica, 26, 1-11. Recuperado de <http://www.revenf.ucr.ac.cr/neurosis.pdf> ISSN 1409-4568

\section{RESUMEN}

El modelo cultural que impera es simplista en cuanto a que imagina el desarrollo de la vida en términos de comienzo, plenitud y decadencia. Durante el envejecimiento, el adulto mayor puede ser testigo de su deterioro físico y psíquico, cambio por la sociedad los califica como personas insuficientes y dependientes, de cuya apreciación se origina una baja autoestima en ellos. Dado lo anterior, esta investigación tiene como principal propósito fomentar la participación de enfermería con el adulto mayor en padecimientos neuróticos para que se tal población se adapte a su problema, independientemente de su naturaleza, y así aumentar su autoestima. El objetivo es proporcionar un soporte integral a los adultos mayores y a sus cuidadores para fortalecer a fin de promover la autoestima de la persona con padecimientos neuróticos para que mantenga su autonomía personal; aparte se procura brindar un soporte integral y de forma continuada a personas dependientes. El planteamiento y desarrollo se fundamenta en la explicación de la patología, de cómo esta afecta el equilibrio de su sistema y cómo ante esta necesidad la enfermería logra que las partes del sistema del cliente interaccionen en armonía con el sistema completo. La finalidad es mostrar cómo se puede lograr un óptimo cuidado de estas personas en colaboración con los familiares, cómo ayudar en el desenvolvimiento de su entorno, aceptando los cambios, y adaptarse e incorporarse a las nuevas circunstancias, mediante el enriquecimiento de su autoestima, mejora que restituirá su bienestar y favorecerá cada ámbito de su vida.

Palabras claves: autoestima, enfermería, envejecimiento, padecimientos-neuróticos

\footnotetext{
${ }^{1}$ Fecha de recepción: 14 de mayo del 2013

Fecha de aceptación: 25 febrero del 2014

${ }^{2}$ Estudiante de la Licenciatura en Enfermería de la Universidad Autónoma de Ciudad Juárez. División Multidisciplinaria Cuauhtémoc.

Correo Electrónico: gioengu 8@,hotmail.com

${ }^{3}$ Docente de la Universidad Autónoma de Ciudad Juárez. Correo electrónico: silvia.najera@uacj.mx
} 


\title{
Nursing: building self-esteem in neurotic disorders in the elderly ${ }^{1}$
}

\author{
Giovanna Enríquez Gutiérrez ${ }^{2}$ \\ Silvia Nájera Aguirre ${ }^{3}$
}

Institution: University of Ciudad Juárez, México.

CITED

Enríquez, G. y Nájera, S. (Abril, 2014) Nursing: building self-esteem in neurotic disorders in the elderly. Rev. Actual de Costa Rica, 26, 1-11.

Recuperado de <http://www.revenf.ucr.ac.cr/neurosis.pdf> ISSN 1409-4568

\begin{abstract}
The prevailing cultural model is simplistic in that it envisions the development of life in terms of beginning, fullness and decay. During aging, older adults can witness their physical and mental deterioration, change society qualifies as poor and dependent, the appreciation of low self-esteem in them arises. Given the above, this research has as main purpose to promote the participation of the elderly with neurotic conditions for such that population fits your problem, regardless of its nature, and increase their self-esteem. The objective is to provide comprehensive support to older adults and their caregivers to strengthen in order to promote self-esteem of people with neurotic condition to maintain personal autonomy; apart seeks to provide a comprehensive and continuously support for dependents. The planning and development is based on the explanation of the disease, how it affects the balance of your system and how to achieve this need and how the parties of the client system interact in harmony with the entire system. The purpose is to show how you can achieve optimal care of these people in collaboration with the family, helping in the development of their environment, accepting the changes, and adapt and incorporate new circumstances by enriching their self- improvement to restore their well-being and promote every area of your life.
\end{abstract}

Keywords: aging, neurotic-suffering, nursing, self-esteem

\footnotetext{
${ }^{1}$ Date of receipt: May 14, 2013

Date of acceptance: february 25, 2014

${ }^{2}$ Student Nursing Degree from the Autonomous University of Ciudad Juárez. Multidisciplinary Division Cuauhtémoc. E mail: gioengu_8@hotmail.com

${ }^{3}$ Professor at the Autonomous University of Ciudad Juárez. E mail: silvia.najera@uacj.mx
} 


\section{INTRODUCCIÓN}

Nuestra sociedad ha experimentado numerosos cambios (en aspectos como la sanidad, mejoría de la alimentación, condiciones de vida, etc.) a partir de los que ha aumentado la esperanza de vida y, como consecuencia, el progresivo envejecimiento de la población. La llamada "tercera edad" es una fase de la vida, marcada por prejuicios que limitan el actuar de la persona adulta mayor, juicio que empobrece la autoestima de esta población.

Una mayor longevidad implica mayor probabilidad de padecer enfermedades propias de esta edad, así como una prolongación de otras existentes. En lo que respecta a las enfermedades del ámbito de la salud mental, algunas de ellas, como la neurosis, combinan en su etiología una cantidad más amplia y variada de factores, al punto de originar un conflicto entre el individuo y su medio, el cual nace en el interior de la persona, quien se niega a aceptar cuanto se dice acerca de este grupo.

En este contexto, la labor del profesional de enfermería es más que significativo, debido a que brinda las herramientas necesarias para proporcionar un soporte integral y holístico a los adultos mayores con padecimientos neuróticos, de manera que se trabaje en su autonomía personal. El abordaje incluye a la familia, debido a que en este espacio es donde se producen cambios, en especial, respecto de las funciones de la persona, así como en las relaciones y la comunicación con el adulto mayor; por tanto, la idea es que la familia y el profesional en enfermería trabajen juntos en función de las necesidades del adulto mayor; no obstante, el paciente deberá adaptarse al intercambio de roles y ello supone una pérdida de su independencia, responsabilidad y liderazgo, lo cual debilitará su autoestima.

Debido a la situación mencionada, es necesario que en este trabajo el profesional de enfermería se informara acerca de diversas posibilidades de intervención que, en este caso, giran alrededor de términos como adulto mayor, padecimiento, neurosis, enfermería, autoestima. Para delimitarlos, se analizaron 62 documentos de los que se seleccionaron tan solo 50 artículos, fundamentados en un modelo de enfermería llamado "modelo de sistemas", de Betty Neuman.

\section{DESARROLLO}

Para iniciar, es imprescindible delimitar el término "enfermedad mental, definido desde diversas ópticas; sin embargo, de acuerdo con el criterio de los autores debe entenderse como el funcionamiento anormal de la psiquis, lo que conlleva una comprensión inadecuada de la realidad y una alteración de los procesos anatómicofisiológicos implicados (Modena, 2005; Lifshitz, 2008).

Una de estas enfermedades mentales, tema de este trabajo, es el padecimiento neurótico, difícil de definir si se tiene plurisintomatología y pluricausalidad; el padecimiento puede estar formado por varias enfermedades simultáneas, por un solo fragmento de la enfermedad o no corresponder a ninguna enfermedad conocida (Modena, 2005; Lifshitz, 2008) 
Debido a las características de los síntomas que presentan los individuos, este problema de salud tiene importantes implicaciones sociales e individuales, sin mencionar la experiencia subjetiva de los síntomas y del sufrimiento; es decir, la experiencia de quien lo padece, así como de quienes rodean a esa persona (Gonzales y Álvarez, 1984; Berenzon y Mora, 2005; Amezcua, 2000; Comas, 2006). Lo anterior significa que el abordaje es integral, dado que se incluyen conceptos, experiencias y sentimientos comunes de un determinado grupo social; se considera elementos de la salud mental y física de la persona, tales como la atención, percepción, respuesta afectiva, cognición, valoración directa ante la enfermedad y sus manifestaciones, así como sus dimensiones culturales (Berenzon, Mora, Torres, 2007; $\underline{\text { Sacchi, Hausberger y Pereyra, 2007; Menéndez, 2005). }}$ )

En cuanto a la neurosis, el término es relativamente joven y fue introducido por el médico escocés W. Cullen, quien la considera una pérdida del sentido del significado de la vida o una radical alteración de la relación con "el otro" (Rivera, Murillo y Sierra, 2007; Thompson y Frydman, 2010).

En la actualidad existe consenso respecto de que no es posible suministrar una definición para neurosis; por consiguiente, puede ser entendida como una perturbación psíquica producida por temores, por la necesidad de protegerse contra estos y por intentos de establecer un equilibrio entre las tendencias en conflicto. Por lo anterior, es correcto afirmar que son disturbios mentales sin una base somática demostrable en los que es indispensable no confundir las experiencias enfermas individuales y sus fantasías con la realidad externa. Los cuadros pueden aparecer de manera periódica o durante toda la vida de la persona y se presentan como episodios leves o como enfermedad grave e incapacitante que, incluso, requiere hospitalización (Toso, 2009; Beltrán y Muñoz 2009; Horney, 2007).

Para comprender mejor el proceso de la neurosis es necesario añadir que se considera consecuencia de un conflicto entre el individuo y su medio, el cual nace de una negación en la persona y se evidencia en una disgregación de las distintas instancias de la personalidad que desembocan en estados de ansiedad, en una desorganización emocional que repercute nocivamente en los procesos psíquicos y en la actividad social de estos enfermos (Sánchez, 2001; Gonzales y Álvarez, 1984; Rodríguez, s.f.).

Dentro del contexto se producen alteraciones en la interpretación y valoración de la realidad a través de varias vías como perturbaciones afectivas dadas por ansiedad, actitudes y jerarquía inadecuada de motivos, mecanismos de defensa, de igual manera están presentes elementos estresantes que son productores de tensión. En cuanto a la etiología común de la enfermedad mental, siempre se menciona la frustración y el incumplimiento de deseos infantiles jamás dominados que se arraigaron hondamente en la mente del individuo que los padece, razón por la que el mecanismo que se activa en la neurosis es no solo una pérdida de la realidad, sino una sustitución de ella. En consecuencia, la neurosis es una expresión de la rebeldía contra el mundo exterior, una incapacidad para adaptarse a la realidad (Sánchez, 2009; Thompson y Frydman, 2010; Rodríguez, s.f.). 
Las investigaciones acerca de las causas originan esta enfermedad concluyen en que mucho de su aparición tiene una raigambre en elementos de la vida sexual. Desde siempre, y por todos los autores, se ha concedido a los factores sexuales cierta relevancia en la etiología de las neurosis; sin embargo, en individuos con una personalidad de rasgos neuróticos se coincide en que el núcleo de las neurosis es la ansiedad . ( $\underline{\text { Campos, }}$ Sotolongo y Santander, 2008; Freud, 1898).

Sin lugar a dudas, existe un creciente interés por el estudio de los trastornos neuróticos, debido no solo a las grandes demandas de atención sanitaria que estos trastornos precisan, sino por la existencia de una gran variedad de tratamientos que pueden resultar efectivos. A lo anterior se une el reconocimiento actual de que son trastornos que impactan a la persona y le generan sufrimiento, repercusiones económicas, aparte de que afectan su funcionamiento, máxime si el envejecimiento se vive como una situación de minusvalía y pérdida, como una manera de marginación social (Sierra, Herran y Vázquez, 2001; Hernández, 2008). Tal como se ha mencionado, el envejecimiento está marcado por cambios físicos y psicológicos, que influyen en nuestra forma de pensar, sentir y de actuar, mas también hay que agregar las transformaciones del mundo que nos rodea, punto de partida para observar y analizar cómo se debe interaccionar en este medio (Vivas y Barrera, 2009).

En el caso de la vejez, una de las causas de que los problemas de neurosis se presenten con tanta frecuencia conforme avanza la edad, se debe a "la dificultad para conciliar el envejecimiento exterior (corporal) con el interior (psicológico). Es un hecho que no siempre la edad biológica y real coinciden, y que este desfase puede, en muchos casos, provocar crisis de identidad" (gentenatural, s.f., s.p.); por ende, es imprescindible procurar la mejor calidad de vida para el adulto mayor, con el fin de prevenir el desarrollo de estos desordenes psíquicos; dicha tarea precisa que el anciano experimente una vida satisfactoria que incluya el bienestar subjetivo y psicológico, su desarrollo personal, así como diversas representaciones de lo que constituye una buena vida y de cómo darle sentido, preservando los valores del contexto en el que vive y la relación con sus propios objetivos de vida (Ordoñez, 2010).

En la actualidad, la neurosis, junto con el cambio en los patrones epidemiológicos de los ancianos ,se centra en problemas crónicos y degenerativos, lo cual propicia que cada vez sea mayor el número de personas que presentan algún tipo de dependencia y que por ello deben recibir cuidados por parte de otros (Escuredo, 2006); en este contexto, el papel de enfermería es básico para proporcionar un soporte integral a las personas con dependencia y a sus cuidadores, promover la permanencia de la persona con dependencias y procurar el mantenimiento de su autonomía personal, así como mejorar la eficacia de los recursos asistenciales destinados a la atención de personas con dependencias y establecer conexiones entre recursos formales e informales (Ubiergo, Regoyos, Vico y Reyes, 2005).

Sin lugar a dudas, la enfermería funge como un sistema de producción de asociaciones entre las personas, parte de la organización del sistema de salud y del sistema de cuidados y área que dialoga con otros sistemas sociales. Para garantizar la eficacia de producción de servicios de enfermería es necesario considerar características como la autonomía, la individualidad, las relaciones y actitudes profesionales y aceptar la 


\section{Revista Electrónica Enfermería Actual en costa Rica}

incertidumbre y la ambivalencia del el sistema de cuidados para enfrentar la complejidad real (Edianez, Micheloto, Perin, Lorenzini y Horner, 2012). El personal de enfermería tiene funciones propias e independientes y se interrelaciona con los miembros del grupo médico, con el que colabora de manera mutua, mas, al mismo tiempo, en un papel de colaboración mutua, así como tiene un papel esencial al gestionar y brindar cuidados al paciente, familia y comunidad de forma holística

El objetivo de la enfermería es proporcionar un sitio seguro para el funcionamiento del sistema cliente y para aislar al cliente de los elementos estresantes, para lo cual cuenta con un acervo relacionado con la disciplina, con una formación que ofrece los conocimientos mínimos para el enfrentamiento de una enfermedad mental. Toda persona con padecimientos mentales requiere cuidado, dado que todo individuo puede cuidar y necesita cuidados; sin embargo, esta capacidad será más o menos desarrollada de acuerdo con las circunstancias y dependerá de la manera como las personas fueron cuidadas durante las etapas de su vida; por consiguiente, factores como el ambiente, la cultura, la economía, la política, la religión, entre otros, interferirán directamente en ese proceso (Edianez, Micheloto, Perin, Lorenzini y Horner, 2012; Otero, 2011; Gutiérrez, Núñez y Rivera, 2002).

La práctica de enfermería va más allá del acto de cuidar que une el "qué" del cuidado y el "cómo" de la interacción persona-enfermera, dado que implica crear un cuidado que recurra a diversos procesos: la reflexión, la integración de creencias y valores, el análisis crítico, la aplicación de conocimientos, el juicio clínico, la intuición, la organización de los recursos y la evaluación de la calidad de las intervenciones. Desde esta perspectiva se trata de un cuidado innovador que une la ciencia y el arte de enfermería y que se centra en la persona, quien vive experiencias de salud en continua interacción con su entorno (Zarate, 2004).

Las enfermeras tienen un papel clave en el cuidado de los ancianos dependientes, ya que su trabajo con las familias les permite tener un conocimiento profundo de los problemas y las necesidades cambiantes de los ancianos y sus cuidadores familiares durante el proceso de dependencia (Escudero, 2006). El cuidado que lleva a cabo la familia se conoce como cuidado familiar o informal y se complementa con los cuidados formales que dependen del sistema sanitario. Los profesionales responsables de esta tarea son las enfermeras que colaboran con las familias en la atención y el cuidado de los dependientes en general y de los ancianos en particular.

Aunado a lo anterior, se ha comprobado que el ser humano en todas las edades, al poseer una buena relación familiar y fuentes de apoyo significativos, se conserva en óptimas condiciones psicológicas y, por ende, supera más fácilmente las tensiones o la propia enfermedad; por el contrario, los adultos mayores con ausencia o mala calidad de estos lazos afectivos carecen de redes de apoyo para superar las pérdidas propias de la edad y los demás problemas que acompañan esta etapa, de modo que el apoyo del profesional de enfermería es invaluable en cuanto a prevenir la aparición de una enfermedad como la neurosis (Sanhueza, Castro y Merino, 2005; Uribe y Valderrama, 2007). 
Dentro de este panorama, la autoestima es esencial para el funcionamiento de la inteligencia emocional: las personas con mejor y más adecuada expresión de sus sentimientos y emociones son a la vez personas seguras de sí, con mayor sentimiento de libertad y autonomía, con mejores relaciones interpersonales y mejor autoestima, lo cual favorece su calidad de vida, debido a que mejora la capacidad funcional y el conjunto de condiciones ideales para fortalecer su autocuidado y la vida familiar y social. La tendencia a favor de una vejez activa procura que el adulto mayor conserve su independencia, su libertad de acción y su iniciativa, de manera que sea capaz de elegir el mayor número de opciones en su vida, sin desconocer la realidad de que la gente de mayor edad es menos capaz de ejercer su independencia, tiene menos eficiencia en su trabajo y es más vulnerable a las enfermedades (Carmenaty y Soler, 2002; Ysern, 2001).

La salud mental es clave para mantener la calidad de vida a lo largo del envejecimiento y gozar satisfactoriamente en la etapa de la vejez, máxime que el individuo puede atravesar por diversas crisis, algunas de tipo circunstancial y otras de desarrollo, que pueden incidir en la salud mental de la persona. Las crisis circunstanciales son aquellas accidentales o inesperadas, mientras que las de desarrollo se relacionan con la transición de una etapa de crecimiento a otra, desde la infancia, hasta la senectud, mas cuando hay interferencia en ese tránsito se produce una crisis. En la medida en que la persona acumule experiencias desagradables desde la infancia, las crisis circunstanciales pueden ser más devastadoras en tanto vienen a reafirmar la autoimagen negativa; no obstante, son también una oportunidad para crecer (Alpízar, 2007).

Por otro lado, la espiritualidad también es parte de la salud mental: una persona que cultiva una adecuada espiritualidad está en capacidad de perdonar y hasta perdonarse, vivir la vida a plenitud, amarse y amar y dar y recibir afecto. En tales individuos resalta la capacidad para el aprovechamiento de los conflictos como oportunidades de crecimiento personal y colectivo, puesto que, desde su sabiduría, afrontan y colaboran con otros en la superación de las dificultades propias de su vida. Muchos autores coinciden en que el comportamiento de las personas mayores se debe no tanto a la edad, sino a reacciones conductuales que reflejan carencias y heridas emocionales sin resolver. Una edad avanzada no es signo de decaimiento o incapacidad intelectual, máxime que este grupo aporta a la sociedad en distintas áreas del conocimiento, de la política, de las artes, sin que importe su edad (Cardona, Estrada y Agudelo ,2005; Alpízar, 2007).

\section{CONCLUSIÓN}

El proceso de vida del ser humano es un proceso dinámico marcado por cambios que, durante el envejecimiento, no solo afectan lo físico, sino también lo psicológico, por ello influyen en la forma de pensar, sentir y actuar. Las transformaciones en la sociedad también deben considerarse, debido a que establecen pautas acerca de cómo observar y analizar el entorno.

Tras la presencia de neurosis en este grupo de edad, el marco de actuación de Enfermería se enfoca en el grupo de adultos mayores con quienes se implementaría estrategias pensadas en mejorar el estado físico mental, social y espiritual de los individuos, mediante la actuación en conjunto con la familia y la comunidad, cuyo fin es evitar la 
aparición de enfermedades psíquicas, procurar mayor bienestar con equidad social y mejorar la calidad de vida. Para desarrollar dicha tarea, la enfermería se vale de los conocimientos aplicados al ejercicio y práctica, concentrándose en la valoración de las necesidades bio-psico-socioculturales y espirituales, así como las intervenciones, acciones determinadas, que ayudan a que el cliente retenga la estabilidad del sistema, establezca medidas a favor de la autoestima y trabaje en su bienestar, superación, equilibrio, afectividad, salud física y mental.

\section{BIBLIOGRAFÍA}

Alpízar, I. (2007) Salud mental y espiritualidad en el envejecimiento y la vejez. II Congreso Internacional de Gerontología (San José, Costa Rica) Recuperado de:

http://gerontología.ucr.ac.cr/web\%20mg/Congreso2/descarga/02Alpizar.pdf

Amezcua, M. (2000). Enfermedad y padecimiento: Significado del enfermar para la práctica de los cuidados. Cultura de los Cuidados. Disponible en http://rua.ua.es/dspace/bitstream/10045/5086/1/CC_07-08 08.pdf

Barrantes, M., Rodríguez, E. y Lama, A. (2009). Relación médico-paciente: derechos del adulto mayor. Acta Bioethica, 15.

Beltrán, J. y Muñoz, M. (2009). Análisis de las teorías de la neurosis en Adler y Jung, a la Luz de las Categorías de Rapaport. Revista Iberoamericana de Psicología: Ciencia y Tecnología, 2(2), 41-48.

Berenzon, S. y Mora, J. (2005). Los padecimientos emocionales cotidianos: percepciones y creencias en dos poblaciones en la Ciudad de México. Investigación en Salud, VII (3), 181-187.

Campos, M., Sotolongo, Y. y Santander, A. (2008). Clínica del estrés. Una modalidad de tratamiento para pacientes neuróticos. Psiquiatría.Com, 12(01). Disponible en:

http://www.psiquiatria.com/revistas/index.php/psiquiatriacom/article/view/227/212/

Canto, H. y Castro, E. (2004). Depresión, autoestima y ansiedad en la tercera edad: un estudio comparativo. Enseñanza e Investigación en Psicología, 9(002), 257-270.

Cardona, D., Estrada, A. y Agudelo, H. (2006). Calidad de vida y condiciones de salud de la población adulta Mayor de Medellín. Biomédica, 26 (2).

Carmenaty, I. y Soler, L. ( 2002). Evaluación funcional del anciano. Revista cubana de Enfermería, 18(3).

Clavijo, A. (2002). Reivindicación de las neurosis. Intersalud. Disponible en: http://Hdl.Handle.Net/10401/2283 
Comas, C. (2006). Lo social del padecimiento subjetivo: un estudio de caso sobre movilidad social disidente. [Tesis para aspirar al grado de Magíster en Ciencia Política y Sociología]. Buenos Aires:. Facultad Latinoamericana de Ciencias Sociales (Flacso).

Edianez, M., Micheloto, G., Perin, G., Lorenzini, A. y Horner, B. (2012). Sistemas de organización de cuidados en Enfermería: reflexiones sobre la complejidad del cuidado como práctica asistencial. Revista Cubana de Enfermería, 28(1)

Escudero, B. (2006). El discurso de las enfermeras ante el cuidado de las personas mayores dependientes y sus cuidadores familiares. Index de Enfermería, 15, 52-53.

Figueredo, N., Sotolongo, I., Arcias, R. y Díaz, G. (2003). Caracterización del adulto mayor en la comunidad. Revista cubana de Enfermería, 19 (3).

Freud, S. (1898). La sexualidad en la etiología de las neurosis. Recuperado de: http://www.biblioteca.org.ar/libros/211761.pdf

Gentenatural (s.f.) Recuperado de: $\underline{\text { www.gentenatural.com }}$

Gonzales, D. y Álvarez, A. (1984). Estudio de la motivación en neuróticos y psicóticos. Revista cubana de Psicología, 1(1).

Gutiérrez, M., Núñez, E. y Rivera, C. (2002). Características del rol en el profesional de enfermería intrahospitalario. Enfermería, 37, 29-33.

Hernández, Y. (2008). Importancia de la debida protección de los derechos humanos del adulto mayor en la sociedad guatemalteca. [Tesis] Guatemala: Universidad de San Carlos de Guatemala.

Horney, K. (2007). La personalidad neurótica de nuestro tiempo. México, D.F.: Paidós.

Hueso, C. (2006). El padecimiento ante la enfermedad. Un enfoque desde la teoría de la representación social. Index de Enfermería, 15(55).

Lifshitz, A. (2008). ¿Padecimiento o enfermedad? Medicina Interna de México, 24(5).

Marriner, A. y Raile, M. (2007). Modelos y teorías en enfermería. Sexta Edición. Madrid: Elsevier.

Menéndez, E. (2005). Modelos de atención de los padecimientos: de exclusiones teóricas y articulaciones prácticas. Diccionario temático Ciesas. Recuperado de:

http://www.ciesas.edu.mx/Publicaciones/diccionario/Diccionario\%20CIESAS/TEMAS\%20PDF/Menendez $\% 207$ $\underline{6 c . p d f}$ 
Modena, M. (2005). Salud-enfermedad-atención y procesos socioculturales. Diccionario temático Ciesas. Recuperado de:

http://www.ciesas.edu.mx/Publicaciones/diccionario/Diccionario\%20CIESAS/TEMAS\%20PDF/Modena\%2079d. pdf

Mordoh, E., Gurevicz, M. y Lombardi, G. (2007). Algunas precisiones sobre el proceso diagnóstico en psicoanálisis. Anuario de Investigaciones, 14.

Moreno, L. (2007). Reflexiones sobre el trayecto salud-padecimiento-enfermedad-atención: una mirada socioantropológica. Salud Pública de México, 49 (1).

Morfi, R. (2005). La salud del adulto mayor en el siglo XXI. Revista cubana de Enfermería, 21(3).

Ordoñez, M. (2010). ¿Histeria, simulación o neurosis de renta? Revista clínica de medicina de familia, 3(1), 3945.

Otero, M. (2011). Salud mental y enfermería. Revista cubana de Enfermería, 27 (1).

Ponente, I. (2007). Salud mental y espiritualidad en el envejecimiento y la vejez. II Congreso Internacional de Gerontología (San José, Costa Rica). Recuperado de:

http://gerontologia.ucr.ac.cr/web\%20mg/Congreso2/descargas/02Alpizar.pdf

Rivera, J., Murillo, J. y Sierra, M. (2007). El concepto de neurosis de William Cullen como revolución científica. Enseñanza e Investigación en Psicología, 12 (001), 157-178.

Rodríguez, S. (s.f.). Eutonía en los ataques de pánico. Disponible en http://www.monografias.com/trabajospdf2/eutonia-ataques-panico/eutonia-ataques-panico.pdf

Sacchi, M., Hausberger, M. y Pereyra, A. (2007). Percepción del proceso salud-enfermedad-atención y aspectos que influyen en la baja utilización del sistema de salud en familias pobres de la ciudad de salta. Salud Colectiva., 3(3), 271-283.

Sánchez, A. (2001). Una aproximación teórica a la relación entre los trastornos neuróticos y los valores éticomorales. Humanidades médicas, 1(2).

Sánchez, A. (2009). Un enfoque axiológico de los trastornos neuróticos. Humanidades médicas, 9 (2).

Sánchez, I. y Pérez, V. (2008). El funcionamiento cognitivo en la vejez: atención y percepción en el adulto mayor. Revista cubana de medicina general integral, 24 (2). 


\section{Revista Electrónica Enfermería Actual en costa Rica}

Sanhueza, M., Castro, M. y Merino, J. (2005). Adultos mayores funcionales. Un nuevo concepto en salud. Ciencia y Enfermería, 11(2), 17-21.

Sierra, D., Herran, A. y Vázquez, J. (2001). Epidemiología y factores de riesgo de los trastornos neuróticos. Psiquiatría.com, 5(4). Recuperado de: http://www.psiquiatría.com/psiquiatria/revista/72/5612

Thompson, S. y Frydman, A. (2010) Acerca de los momentos electivos en la producción y tratamiento de la neurosis. Anuario de Investigaciones, 17.

Torres, T. (2007). Una aproximación cualitativa a estudio de las enfermedades crónicas: las representaciones sociales. Ciencias Sociales y de la Salud. Recuperado de:

http://www.cucs.udg.mx/iiso/files/File/101\%20Una $\% 20$ aproximacion $\% 20$ cualitativa $\% 20 \mathrm{al} \% 20$ estudio $\% 20 \mathrm{de} \% 2$ 0las $\% 20$ enf $\% 20$ cronicas.pdf

Toso, M. (2009). Neurosis: desequilibrio psicológico, estrés y sistemas somáticos. Recuperado de: http://es.shvoong.com/medicine-and-health/epidemiology-public-health/1928567-neurosis-

desequilibriopsicol\%c3\%b3gico-estr\%c3\%a9s s\%c3\%adntomas/\#ixzz24vufoi9t

Trejo, C. (2001). El viejo en la historia. Acta Bioethica, 7 (01).

Ubiergo, M., Regoyos, S., Vico, M. y Reyes, R. (2005). El soporte de enfermería y la claudicación del cuidador informal. Enfermería clínica, 15 (4), 199-205.

Uribe, A. y Valderrama, L. (2007). Actitud y miedo ante la muerte en adultos mayores. Pensamiento psicológico, $3(8), 109-120$.

Uribe, A., Valderama, L. y Molina, J. (2007). Salud objetiva y salud psíquica en adultos mayores colombianos. Acta colombiana de Psicología, 10 (001), 75-81.

Villanueva, L. (2002). Sobre el envejecimiento: una perspectiva integral. Rev. Hosp. Gral. Dr. M Gea González., 3 (3), 107-114. Recuperado de: http:/www.medigraphic.com/pdfs/h-gea/gg-2000/gg003d.pdf

Vivas, M. y Barrera, W. (2009). Mejoramiento de la afectividad del adulto mayor: buscando la autorrealización personal en el grupo edad de oro. Caloto - Cauca. Recuperado de: http://repository.unad.edu.co/eprints/bitstream/10597/531/1/2009-76p-22.pdf

Ysern de Arce, J. (2001). Avances y desafíos para un enfoque integral. Recuperado de: http://www.redadultosmayores.com.ar/docspdf/regiones/mercosur/brasil/07inteligenciaemocionalenelam.pdf

Zárate, R. (2004). La gestión del cuidado de enfermería. Index de Enfermería, 13, 44-45. 


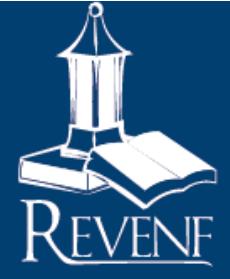

Revista Electrónica Enfermería Actual en costa Rica

Zavala, M., Vidal, D., Castro, M., Quiroga, P. y Klassen, G. (2006). Funcionamiento social del adulto mayor. Ciencia y enfermería, 12 (2), 53-62. 